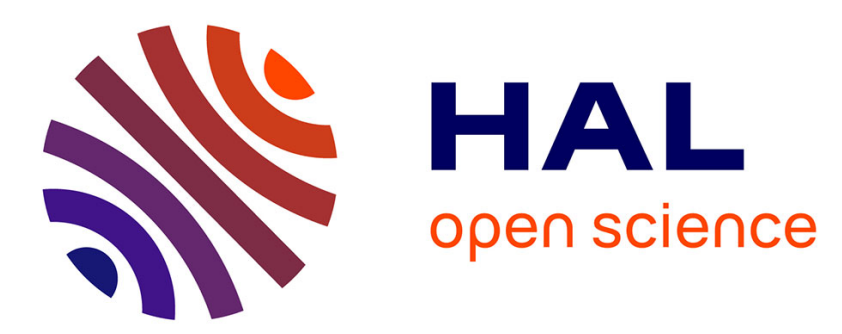

\title{
Stratégies logistiques émergentes : de la grande distribution alimentaire aux réseaux contractuels
}

Odile Chanut, Gilles Paché

\section{To cite this version:}

Odile Chanut, Gilles Paché. Stratégies logistiques émergentes : de la grande distribution alimentaire aux réseaux contractuels. Marché et Organisations, 2012, 15 (1), 10.3917/maorg.015.0091 . hal01767220

\section{HAL Id: hal-01767220 \\ https://hal.science/hal-01767220}

Submitted on 17 Apr 2018

HAL is a multi-disciplinary open access archive for the deposit and dissemination of scientific research documents, whether they are published or not. The documents may come from teaching and research institutions in France or abroad, or from public or private research centers.
L'archive ouverte pluridisciplinaire HAL, est destinée au dépôt et à la diffusion de documents scientifiques de niveau recherche, publiés ou non, émanant des établissements d'enseignement et de recherche français ou étrangers, des laboratoires publics ou privés. 


\section{STRATÉGIES LOGISTIQUES ÉMERGENTES : DE LA GRANDE DISTRIBUTION ALIMENTAIRE AUX RÉSEAUX CONTRACTUELS}

Odile Chanut, Gilles Paché

\section{L'Harmattan | « Marché et organisations »}

2012/1 N 15 | pages 91 à 115

ISSN 1953-6119

ISBN 9782296563117

Article disponible en ligne à l'adresse :

https://www.cairn.info/revue-marche-et-organisations-2012-1-page-91.htm

\section{Pour citer cet article :}

Odile Chanut, Gilles Paché« Stratégies logistiques émergentes : de la grande distribution alimentaire aux réseaux contractuels », Marché et organisations 2012/1 $\left(\mathrm{N}^{\circ} 15\right)$, p. 91-115.

DOI 10.3917/maorg.015.0091

Distribution électronique Cairn.info pour L'Harmattan.

(C) L'Harmattan. Tous droits réservés pour tous pays.

La reproduction ou représentation de cet article, notamment par photocopie, n'est autorisée que dans les limites des conditions générales d'utilisation du site ou, le cas échéant, des conditions générales de la licence souscrite par votre établissement. Toute autre reproduction ou représentation, en tout ou partie, sous quelque forme et de quelque manière que ce soit, est interdite sauf accord préalable et écrit de l'éditeur, en dehors des cas prévus par la législation en vigueur en France. Il est précisé que son stockage dans une base de données est également interdit. 


\section{STRATÉGIES LOGISTIQUES ÉMERGENTES : DE LA GRANDE DISTRIBUTION ALIMENTAIRE AUX RÉSEAUX CONTRACTUELS}

\section{Odile CHANUT Gilles PACHÉ}

Un article publié dans Supply Chain Magazine de janvier 2010 relate une expérience de mutualisation des ressources logistiques particulièrement symptomatique de stratégies collectives actuellement émergentes en France. Ainsi, quinze entreprises du Finistère ont décidé de se regrouper pour réduire leurs coûts de transport, alors que les grands distributeurs exigent de leur part des livraisons de plus en plus fractionnées dans le cadre d'une politique d'approvisionnement en juste-àtemps (JAT). Suite à un appel d'offres lancé en octobre 2009, le cabinet Supply Chain Masters a été sélectionné pour conduire l'étude de faisabilité d'une mutualisation ayant finalement abouti fin 2010. Le directeur associé de Supply Chain Masters se félicite de cette initiative : "Guidée par les principes du développement durable, cette action collective a pour objectif de trouver de nouveaux gisements de croissance et de productivité par la mise en réseau des supply chains des PME ». Le projet consistait à évaluer différents scénarii de mutualisation susceptibles d'améliorer la compétitivité des entreprises, et de diminuer les kilomètres parcourus ainsi que les émissions de $\mathrm{CO}_{2}$. En bref, l'heure est venue de penser des actions 
concertées plutôt qu'individuelles, et l'exemple cité n'est qu'une réalisation parmi de nombreuses autres.

Pour aborder la question de la mutualisation, nous nous réfèrerons à la définition qu'en donne le PIPAME (2009, p. 38) : «La mutualisation en transport et logistique est un accord de partenariat qui consiste à la mise en commun volontariste de moyens physiques, d'informations et de compétences dans le but d'obtenir à long terme des gains économiques, écologiques, financiers et/ou d'image, ou bien encore, de parer à court terme à une contrainte. Le cadre de cette coopération peut revêtir des formes juridiques et organisationnelles variables en fonction de la nature des parties, des moyens et des produits ou services. " Si l'on parle de mutualisation des ressources logistiques de manière récurrente depuis quelques années, il faut admettre que les grands distributeurs ont compris rapidement, dès les années 1980 , l'intérêt de partager des capacités de transport ou de stockage en recourant notamment à des prestataires de services logistiques (PSL) communs. Ce partage est une source d'avantage concurrentiel dans la mesure où il permet à la grande distribution, alimentaire ou spécialisée (GDA, GSS), de bénéficier d'économies d'échelle, et d'optimiser l'usage de ressources logistiques rares.

Les démarches de mutualisation connaissent toutefois aujourd'hui une réelle accélération, et la généralisation de leur portée sous l'effet de l'urgence sociétale du développement durable. Plus précisément, les démarches de mutualisation inscrivent l'action des entreprises, y compris en concurrence frontale, dans un projet politique global, illustré en France par le Grenelle de l'Environnement. Sur cette base, l'objectif de l'article est d'examiner si les démarches de mutualisation initiées par la grande distribution dès les années 1980 constituent une sorte de modèle standard, pouvant s'appliquer à d'autres secteurs d'activité.

Le cas des réseaux contractuels, et singulièrement des réseaux de franchise, est ici retenu pour une exploration du caractère universel ou non des démarches de mutualisation. Dans les réseaux contractuels, comme dans la GDA, la logistique est perçue, depuis de nombreuses années, comme une source d'avantage concurrentiel. Venant en soutien du processus de centralisation des activités d'achat, elle est organisée par le 
franchiseur pour optimiser la livraison vers les magasins de son réseau, et ainsi réduire les coûts de transport et de stockage, tout en améliorant la qualité de service rendue. De ce point de vue, il est possible de parler de mutualisation verticale entre membres d'un réseau liés par des solidarités en termes d'image, d'offre marketing mais aussi de performance économique et financière. Au-delà, la logistique des réseaux contractuels doit désormais tenir compte de nouveaux enjeux économiques et environnementaux qui pourraient conduire les acteurs à emprunter la voie d'une mutualisation horizontale entre réseaux contractuels distincts, mais intervenant dans une même aire urbaine, et donc liés par des solidarités en termes d'optimisation logistique.

En effet, si l'objectif de réduction des coûts reste présent dans les réseaux contractuels, avec les hausses récurrentes du prix de l'énergie, des pressions environnementales ne cessent de s'ajouter, sous l'influence de populations sensibilisées aux effets néfastes des activités logistiques dans la ville. La réduction du nombre de kilomètres parcourus et l'optimisation du remplissage des camions deviennent ainsi des priorités en vue de réduire les émissions de $\mathrm{CO}_{2}$. Sachant que les différents réseaux contractuels se retrouvent souvent dans les mêmes rues des centres ville, du fait de stratégies d'implantation proches (Chanut et Boulay, 2010), les processus de livraison pourraient être repensés de manière à approvisionner simultanément des réseaux contractuels concurrents d'un même territoire, plutôt que les magasins d'un même réseau contractuel sur tout un pays. L'article examine si les expériences réussies de mutualisation des ressources logistiques entre concurrents dans la GDA, en tant que stratégies émergentes, sont imitables ou non par les réseaux contractuels, et à quelles conditions.

\section{LOGISTIQUE DES DISTRIBUTEURS : UNE ÉVOLUTION EN QUATRE ÉTAPES}

Il est désormais admis que la logistique est au cœur des stratégies des industriels et des grands distributeurs alimentaires et spécialisés. D'une vision purement opérationnelle, fondée sur l'optimisation des activités liées à l'acheminement des produits 
finis de l'usine jusqu'aux linéaires des magasins et/ou au retour des produits invendus ou en fin de vie, la logistique a vu émerger au début des années 1990 une vision plus intégrative. Elle repose sur une démarche de management capable de faire travailler étroitement et durablement un ensemble d'entreprises (fournisseurs, assembleurs, grands distributeurs, PSL) afin de créer en commun de la valeur pour le client (Paché, 2004). Une sémantique nouvelle accompagne le changement de perspective puisque l'on parle désormais de « solidarités de chaîne " et de supply chain management. La compétition s'est déplacée pour opposer non plus les entreprises entre elles (Procter \& Gamble vs Unilever), mais les chaînes logistiques entre elles, pour reprendre l'analyse de Christopher (2010).

\section{Trois modèles explicatifs d'un processus majeur de reconfiguration}

Trois modèles logistiques se sont succédé pour répondre aux évolutions de la GDA et de la GSS depuis quarante ans. Lors du décollage des formats supermarché et hypermarché, dans les années 1960 et 1970, le modèle logistique dominant était celui des livraisons directes des usines aux magasins (modèle logistique 1). Les coûts logistiques étaient alors supportés par les industriels, marginalement par les grossistes. Avec la multiplication du nombre de grandes surfaces, leur course à la taille critique et la hausse du nombre de références présentes en linéaire, les grands distributeurs ont été amenés à créer des centres de distribution (CD) dans les années 1980, sous forme d'entrepôts et/ou de plates-formes. C'est la naissance du modèle logistique 2, celui des livraisons des usines vers les CD des grands distributeurs, ceux-ci étant ensuite chargés d'approvisionner leurs magasins. Les CD sont gérés directement par les distributeurs ou par l'intermédiaire de PSL qui finissent d'ailleurs par prendre en charge des tâches de plus en plus nombreuses et complexes (gestion des commandes, co-packing, comanufacturing, etc.).

Sur le plan organisationnel et opérationnel, le modèle logistique 2 conduit à mutualiser les stocks d'un même grand distributeur dans un ou plusieurs CD et de réduire d'autant les réserves en magasin. Ces dernières sont progressivement transfor- 
mées en surfaces de vente, notamment lorsque la Loi Raffarin (juillet 1996) soumet à autorisation administrative toute création ou agrandissement de surface de vente supérieure à $300 \mathrm{~m}^{2}$, rendant donc plus rares les $\mathrm{m}^{2}$ consacrés à la vente. Les années 1990 conduisent à la multiplication des CD des grands distributeurs, dont la taille croissante ne permet pas toujours un fonctionnement au maximum de leurs capacités ; la raison en est la création d'infrastructures logistiques par chaque enseigne, sans aucune concertation avec ses concurrents. Il faudra attendre le milieu des années 2000 pour qu'un nouveau modèle logistique émerge timidement, avec l'implantation en amont des CD des grands distributeurs d'entrepôts multi-producteurs, partagés par plusieurs industriels. Le modèle logistique 3 marque une évolution vers la mutualisation, mais uniquement dans le cadre d'un unique grand distributeur (Hiesse et Paché, 2010). L'idée n'est donc pas de mettre en commun des ressources et compétences logistiques entre plusieurs grands distributeurs, mais d'optimiser les livraisons venant des industriels, celles des PME notamment, en les groupant en direction des CD du grand distributeur.

Si la première finalité de la logistique a été l'amélioration de la performance (réduction des coûts, amélioration de la qualité de service, réactivité accrue) et la création d'un avantage concurrentiel pour les grands distributeurs, d'autres enjeux se sont ajoutés au fil du temps. La maîtrise des infrastructures logistiques est ainsi devenue une arme dans la compétition verticale au sein du canal de distribution. Le coût logistique, qui représente encore 6 à $8 \%$ des prix de vente des biens de grande consommation en moyenne, est en partie transféré de l'industriel vers le grand distributeur, mais la compensation financière demandée aux industriels dépasse largement l'économie de coût réalisée. Par ailleurs, les informations stratégiques liées aux comportements des consommateurs sont désormais détenues par les grands distributeurs, ce qui accroît leur pouvoir de négociation face aux industriels. Maîtriser la logistique devient ainsi une démarche de knowledge management, et n'est plus cantonné au meilleur pilotage des flux en vue de satisfaire la demande (Rajesh et al., 2011).

Une troisième série d'enjeux émerge plus récemment, pour faire face aux mutations en cours qui exigent une utilisation 
durable des ressources logistiques. Des réponses structurées sont indispensables pour relever le défi de l'urgence sociétale $\mathrm{du}$ développement durable. Les initiatives de «logistique verte » commencent à se multiplier, tant il est vrai que les activités logistiques sont justement à l'origine de nombreuses dégradations environnementales. Parmi elles, la mutualisation des ressources logistiques occupe une place importante à la fois chez les décideurs politiques, les acteurs des collectivités territoriales et les managers d'entreprise. En bref, mutualiser consiste à organiser, de manière volontaire et structurée, un partage de ressources entre entreprises, qui peuvent par ailleurs être frontalement concurrentes sur le marché. La contribution récente de Pan et al. (2011) souligne ainsi l'impact d'une mutualisation réussie sur les émissions de $\mathrm{CO}_{2}$ grâce à une gestion efficace d'un mix transport routier/transport ferroviaire. Pour relever les défis environnementaux, un nouveau modèle semble se concrétiser chez les grands distributeurs, en continuité avec les trois précédents.

\section{Vers l'émergence d'un quatrième modèle logistique?}

L'idée de mutualisation n'est pas révolutionnaire puisqu'elle trouve ses racines dans le recours à des PSL communs à plusieurs chaînes logistiques dès les années 1980, et de manière systématique à partir des années 1990 (Albertini, 2008). En revanche, la nouveauté consiste à penser la mutualisation comme une manœuvre collective incontournable, au service d'objectifs sociétaux, et à considérer qu'elle s'impose aux stratégies individuelles d'entreprise. Les expériences de mutualisation peuvent être qualifiées de verticales lorsqu'elles aboutissent à la mise en commun de moyens entre entreprises situées à des niveaux différents du canal de distribution. Elles peuvent être qualifiées d'horizontales lorsqu'elles impliquent des entreprises ou groupes d'entreprises directement concurrentes au même stade de la chaîne de production-distribution. Les deux niveaux de mutualisation, vertical et horizontal, peuvent toutefois être combinés.

Une parfaite illustration du possible enchevêtrement des différents niveaux de mutualisation est celle de l'entrepôt mutualisé que gère le prestataire ID Logistics sur la zone Clésud de 
Miramas (Bouches-du-Rhône), depuis mars 2011, pour le compte de Carrefour et de Danone Eaux France. ID Logistics dispose de $40000 \mathrm{~m}^{2}$ de surfaces d'entreposage dédiées aux produits Danone Eaux France (marques Volvic et Evian) en vue de leur livraison sur les 35 hypermarchés Carrefour et les 80 supermarchés Carrefour Market du Sud de la France. Mais l'entrepôt mutualisé, approvisionné par transport ferroviaire, sert également de plate-forme de proximité pour d'autres enseignes de la région, pourtant directement concurrentes de Carrefour, selon une logique de mutualisation horizontale. L'utilisation $\mathrm{du}$ fret ferroviaire doit réduire d'environ 500 tonnes l'empreinte carbone en 2011, et de $25 \%$ les émissions de $\mathrm{CO}_{2}$ sur toute la zone Sud-Est. Les usines d'embouteillage d'Evian et de Volvic livrant l'entrepôt mutualisé en trains complets, ce sont en effet près de 1000 camions par an qui n'emprunteront plus les axes routiers (un train complet équivaut à 40 camions).

L'entrepôt mutualisé de Clésud à Miramas n'est pas qu'une expérience isolée. Il correspond au contraire à une évolution lourde des politiques d'entreprises, impulsée notamment par le Club Demeter, association créée sur l'initiative de responsables et de chefs d'entreprise des acteurs de la filière agroalimentaire pour anticiper et préparer les mutations à venir en matière de logistique et de développement durable. Les mutualisations verticale et horizontale constituent, de ce point de vue, la manifestation de profondes ruptures dans le pilotage des flux, qui vont avoir des conséquences à de multiples niveaux. Deux aspects peuvent plus particulièrement retenir l'attention :

1) La capacité à conduire dans la durée des stratégies coopétitives gagnantes. Quoi de plus paradoxal que d'imaginer des entreprises en compétition, ayant patiemment construit une organisation logistique pour se démarquer de leurs concurrents, et qui acceptent finalement de partager avec eux des ressources dans une logique de mutualisation ? L'urgence sociétale s'avère pourtant désormais très présente, elle ne pourra empêcher une telle mutation dans le fonctionnement des chaînes logistiques. La traduction en est une généralisation rapide des stratégies coopétitives ; autrement dit, les entreprises devront apprendre " chemin faisant » à gérer entre elles une situation de compétition frontale sur certaines dimensions de leur chaîne de valeur, tout en coopérant sur d'autres dimensions (Yami et Le Roy, 
2010). Une telle démarche de coopération, couplée à une concurrence directe sur le marché pour conquérir le consommateur, a souvent été conduite à un échelon que l'on peut qualifier d'horizontal, par exemple dans la mise au point d'emballages communs (entre les géants Coca-Cola et Pepsi-Cola). L'extension au domaine de la logistique apparaît donc naturelle mais pose de redoutables problèmes dans la durée. En effet, le pilotage des flux permet d'accéder à des données stratégiques sur les ventes réalisées, les opérations promotionnelles en cours, etc., et la mutualisation offre de fait un accès en partie transparent à ces informations stratégiques pour tous les partenaires d'une chaîne logistique. Concilier coopération et compétition en contexte de mutualisation oblige à reformuler la gouvernance des chaînes logistiques, sur un modèle stratégique radicalement nouveau.

2) L'intervention régulatrice des autorités locales. Si l'on adopte une perspective ouverte sur les interactions entre action privée et interventionnisme public, il faut admettre que la mutualisation des ressources logistiques s'inscrit parfaitement dans le projet d'une « ville durable », soucieux d'éviter la saturation et la pollution des espaces de vie faute d'une coordination suffisante des flux de marchandises. Par exemple, la principauté de Monaco tente de mettre en œuvre un système planifié de livraisons urbaines à partir d'un entrepôt localisé à $30 \mathrm{~km}$ de là, en décourageant les approvisionnements directs (et anarchiques) vers les magasins. L'idée est d'organiser des tournées mutualisées à partir de l'entrepôt, fondées sur le groupage des produits à destination des différents magasins ${ }^{1}$. De telles expérimentations se multiplient en Allemagne autour du concept de City Logistik, et aboutissent à une intervention de plus en plus importante des autorités locales dans la gestion des opérations de logistique urbaine (Paché, 2010).

En bref, un modèle logistique 4 émerge, qui associe trois organisations logistiques: dans un premier temps, les industriels livrent un entrepôt multi-producteurs (aussi appelé entre-

\footnotetext{
${ }^{1}$ Force est cependant de reconnaître, en dehors de l'existence de tout cadre coercitif, que les livraisons directes restent encore dominantes pour les grandes surfaces alimentaires monégasques, comme le confirme le Directeur général du Supermarché Casino localisé sur le port de Monaco (entretien réalisé par Odile Chanut le 2 mars 2011).
} 
pôt primaire) au sein duquel les flux sont massifiés ; dans un second temps, les industriels livrent - à plusieurs - les entrepôts de grands distributeurs (aussi appelés entrepôts secondaires) en fonction de leurs besoins. Ces entrepôts livrent, dans un troisième temps les magasins, ou les centres de dégroupage en périphérie des villes (aussi appelés entrepôts de consolidation). Les stratégies actuelles de mutualisation des ressources logistiques conduisent ainsi à repenser les processus clé de la chaîne logistique et l'affectation des ressources entre entreprises. La GDA, comme l'indique le cas du partenariat entre Carrefour et Danone Eaux France, est à l'origine de modèles de management bien établis et jugés très efficaces. Il en ressort des principes que l'on pourrait imaginer universels dans l'organisation de la mutualisation des ressources logistiques (création de centres de consolidation indépendants par chacun des grands distributeurs, partage des ressources de transport entre magasins d'un même territoire, etc.). Est-ce véritablement le cas ?

\section{Tableau 1 : Enjeux, structures et parties prenantes de la logistique des grands distributeurs en France}

\begin{tabular}{|c|c|c|}
\hline & Années 1970 & Années 1980 \\
\hline $\begin{array}{l}\text { Évolution des } \\
\text { structures de } \\
\text { distribution }\end{array}$ & $\begin{array}{l}\text { Décollage des formats } \\
\text { modernes de distribution } \\
\text { (super et hypermarché) }\end{array}$ & $\begin{array}{l}\text { Course à la taille et } \\
\text { hausse du nombre de } \\
\text { références et de } \\
\text { magasins }\end{array}$ \\
\hline $\begin{array}{l}\text { Évolution des } \\
\text { structures } \\
\text { logistiques }\end{array}$ & $\begin{array}{l}\text { Modèle } 1 \\
\text { Livraison directe des usines } \\
\text { aux magasins des grands } \\
\text { distributeurs }\end{array}$ & $\begin{array}{l}\text { Modèle } 2 \\
\text { Livraison des usines } \\
\text { aux CD des grands } \\
\text { distributeurs }\end{array}$ \\
\hline $\begin{array}{l}\text { Enjeux } \\
\text { stratégiques de } \\
\text { la logistique }\end{array}$ & $\begin{array}{l}\text { En termes de passage d'une } \\
\text { économie de l'offre à une } \\
\text { économie de la demande : } \\
\text { - pratiques de JAT } \\
\text { - point de découplage }\end{array}$ & $\begin{array}{l}\text { En termes d'avantages } \\
\text { concurrentiels : } \\
\text { - baisse des coûts } \\
\text { (transfert des coûts de } \\
\text { l'industriel au grand } \\
\text { distributeur) } \\
\text { - niveaux de service } \\
\text { croissants (réduction } \\
\text { des ruptures, livraisons } \\
\text { plus fréquentes) }\end{array}$ \\
\hline $\begin{array}{l}\text { Principaux } \\
\text { acteurs de la } \\
\text { logistique }\end{array}$ & $\begin{array}{l}\text { Industriels } \\
\text { Grands distributeurs }\end{array}$ & $\begin{array}{l}\text { Industriels } \\
\text { Grands distributeurs }\end{array}$ \\
\hline
\end{tabular}




\begin{tabular}{|c|c|c|}
\hline & & $\begin{array}{l}\text { PSL de première } \\
\text { génération }\end{array}$ \\
\hline \multirow[t]{2}{*}{ Mutualisation } & & $\begin{array}{l}\ldots \text { des stocks des } \\
\text { magasins (réduction } \\
\text { des réserves) }\end{array}$ \\
\hline & $1990-2005$ & Depuis 2005 \\
\hline $\begin{array}{l}\text { Évolution des } \\
\text { structures de } \\
\text { distribution }\end{array}$ & $\begin{array}{l}\text { Parc de magasins à } \\
\text { maturité, Loi Raffarin } \\
\text { (rareté des surfaces de } \\
\text { vente) }\end{array}$ & $\begin{array}{l}\text { Retour des magasins } \\
\text { de proximité } \\
\text { Développement du } \\
\text { e-commerce et des } \\
\text { livraisons à domicile }\end{array}$ \\
\hline $\begin{array}{l}\text { Évolution des } \\
\text { structures } \\
\text { logistiques }\end{array}$ & $\begin{array}{l}\text { Modèle } 2 \text { (suite) } \\
\text { Dissémination et } \\
\text { spécialisation des CD des } \\
\text { grands distributeurs } \\
\text { Essor du supply chain } \\
\text { management avec création } \\
\text { d'outils collaboratifs } \\
\text { (GMA, CPFR, etc.) }\end{array}$ & $\begin{array}{l}\text { Modèle } 3 \\
\text { Livraison des usines } \\
\text { vers des entrepôts } \\
\text { multi-producteurs puis } \\
\text { vers les CD des grands } \\
\text { distributeurs } \\
\text { Modèle } 4 \\
\text { Trois organisations } \\
\text { logistiques } \\
\text { Vers la création de } \\
\text { centres de dégroupage } \\
\text { en périphérie des } \\
\text { villes? }\end{array}$ \\
\hline $\begin{array}{l}\text { Enjeux } \\
\text { stratégiques de } \\
\text { la logistique }\end{array}$ & $\begin{array}{l}\text { En termes de jeux de } \\
\text { pouvoir: } \\
\text { - intérêt de contrôler la } \\
\text { chaîne logistique } \\
\text { - connaissance des marchés, } \\
\text { partage négocié de l'infor- } \\
\text { mation sur la demande }\end{array}$ & $\begin{array}{l}\text { En termes de déve- } \\
\text { loppement durable : } \\
\text { - baisse du nombre de } \\
\mathrm{km} \text { parcourus et impact } \\
\mathrm{CO}_{2} \\
\text { - fluidité de la circula- } \\
\text { tion en centre ville } \\
\text { - hausse des fréquences } \\
\text { de livraison } \\
\text { - recherche d'alterna- } \\
\text { tives au transport } \\
\text { routier (fer, fluvial) }\end{array}$ \\
\hline $\begin{array}{l}\text { Principaux } \\
\text { acteurs de la } \\
\text { logistique }\end{array}$ & $\begin{array}{l}\text { Industriels } \\
\text { Grands distributeurs } \\
\text { PSL de deuxième } \\
\text { génération (complexifi- } \\
\text { cation du package de } \\
\text { services) }\end{array}$ & $\begin{array}{l}\text { Industriels } \\
\text { Grands distributeurs } \\
\text { PSL de deuxième } \\
\text { génération (complexi- } \\
\text { fication du package de } \\
\text { services) }\end{array}$ \\
\hline
\end{tabular}




\begin{tabular}{|c|c|c|}
\hline & & $\begin{array}{l}\text { Autorités locales } \\
\text { Pouvoirs publics }\end{array}$ \\
\hline Mutualisation & $\begin{array}{l}\text {.. des transports, avec un } \\
\text { PSL comme vecteur de } \\
\text { mutualisation }\end{array}$ & $\begin{array}{l}\text {... des entrepôts entre } \\
\text { industriels } \\
\ldots \text { des CD entre grands } \\
\text { distributeurs? }\end{array}$ \\
\hline
\end{tabular}

Dans les faits, les réflexions logistiques et le supply chain management ont concerné en premier lieu les structures de taille importante, avec des initiatives séparées et/ou conjointes menées par des industriels et des grands distributeurs. Une telle réalité ne peut être contestée, elle occupe d'ailleurs la majorité des travaux consacrés au thème de la mutualisation des ressources logistiques (Hiesse et Paché, 2010 ; Pan, 2010). Mais comment en va-t-il pour des structures organisationnelles n'ayant pas la même envergure, et ne pouvant donc bénéficier d'économies de champ significatives ? La question est tout particulièrement pertinente pour les réseaux contractuels, qui sont loin de disposer de ressources et compétences logistiques comparables à celles de la GDA. Autrement dit, les évolutions constatées dans la GDA vers plus de mutualisation verticale, puis horizontale, sont-elles finalement constitutives d'un invariant à portée générale ? C'est l'interrogation centrale examinée dans la deuxième partie de l'article.

\section{QUELS ENJEUX LOGISTIQUES POUR LES RÉSEAUX CONTRACTUELS ?}

Les réseaux contractuels, et notamment les réseaux de franchise, sont devenus en quelques années un phénomène économique incontournable du paysage commercial dans de nombreux pays occidentaux (Boulay et Chanut, 2010). Ils ont effectivement envahi les centres commerciaux et les centres ville, et rares sont les rues piétonnes où des enseignes comme Pizza Hut, Yves Rocher, De Neuville, Quick ou Monceau Fleurs sont absents. Uniquement pour la franchise, on comptabilise en France, début 2011, plus de 1470 réseaux contractuels et 58000 magasins, dans les secteurs d'activité les plus variés, pour distribuer des produits ou des services au consommateur. 
Tableau 2 : La franchise en France : quelques chiffres clé

\begin{tabular}{|c|c|c|c|}
\hline & $2001^{*}$ & $2005^{*}$ & $2010 *$ \\
\hline $\begin{array}{l}\text { Nombre de réseaux } \\
\text { franchisés }\end{array}$ & 653 & 929 & 1477 \\
\hline $\begin{array}{l}\text { Nombre de magasins } \\
\text { franchisés }\end{array}$ & 32240 & 39510 & 58346 \\
\hline $\begin{array}{l}\text { CA de la franchise } \\
\text { (milliards } € \text { ) }\end{array}$ & 30,5 & 43 & 47,9 \\
\hline $\begin{array}{l}\text { La répartition par } \\
\text { secteur des unités } \\
\text { franchisées** } \\
\text { (en \% des unités } \\
\text { franchisées) }\end{array}$ & \multicolumn{3}{|c|}{$\begin{array}{l}\text { Alimentaire }(15,2) \text {; Équipement de la personne } \\
(14) \text {; Commerce divers }(11,2) \text {; Coiffure et } \\
\text { esthétique }(10,7) \text {; Hôtellerie-restauration }(10,1) \text {; } \\
\text { Services auto }(9) \text {; Immobilier }(8,6) \text {; Équipement } \\
\text { de la maison }(7,2) \text {; Autres services aux } \\
\text { personnes }(6,9) \text {; Services aux entreprises }(2,4) \text {; } \\
\text { Bâtiment }(2,1) \text {; Voyages }(1,1) \text {; Nettoyage }(1) ; \\
\text { Formation }(0,5)\end{array}$} \\
\hline $\begin{array}{l}\text { Exemples d'enseignes } \\
\text { franchisées }\end{array}$ & \multicolumn{3}{|c|}{$\begin{array}{l}\text { Adhap Services, But, Cannelle, Carré Blanc, } \\
\text { Cartridge World, Casa, Cavavin, Century 21, } \\
\text { Class'Croûte, Clair de Baie, Domino's Pizza, } \\
\text { Ecotel, Etam, Epil Center, Etap Hotel, Europcar } \\
\text { International, Feu Vert, Fly, France Loisirs, } \\
\text { FNAC Services, Foir'Fouille, Générale } \\
\text { d'Optique, Glup's, Havas Voyage, Histoire de } \\
\text { Pains, Ibis, Irrijardin, Jacadi, Jacqueline Riu, Le } \\
\text { Jardin des Fleurs, Jeff de Bruges, Jules, Kyriad, } \\
\text { McDonald's, Mercure, Midas, Monceau Fleurs, } \\
\text { Happy, Olly Gan, Pomme de Pain, Orchestra, } \\
\text { Physiomins, Phildar, Pizza Hut, Point Chaud, } \\
\text { Quick Restaurant, Saint Algues Coiffure, Sign A } \\
\text { Rama, Soho, Spar, Speedy, Le Tanneur, Troc de } \\
\text { l'Ile, Triba, Ucar, La Vie Claire, Yves Rocher, } \\
\text { etc. }\end{array}$} \\
\hline
\end{tabular}

* Chiffres au 31 décembre de l'année (Source : Fédération française de la franchise);** Données début 2010.

$\mathrm{Au}$ total, la franchise génère 315000 emplois directs et 725000 emplois indirects ${ }^{1}$. Ajoutons qu'elle tient chaque année Salon à Pékin, à Shanghai, à Moscou, à Kuala Lumpur ou au Caire, attirant un nombre toujours plus important d'individus désireux de devenir leur propre patron. Le succès des réseaux contractuels ne peut donc se réduire, une fois encore, à une

\footnotetext{
${ }^{1}$ Selon des données fournies par la Fédération française de la franchise.
} 
« exception française »; la situation américaine témoigne au demeurant d'une dynamique économique que l'on peut qualifier de globale (LaFontaine, 2009).

\section{Une forme organisationnelle originale}

Il existe plusieurs types de contrats dans les réseaux contractuels, qui diffèrent sur certains points mais relèvent de la même logique : partage des investissements, des risques et des profits entre une tête de réseau, agissant comme centre stratégique de la relation ou pivot (Assens, 2003), et des commerçants indépendants, propriétaires de leurs fonds de commerce. Les contrats de concession, courants dans l'automobile ou chez les cuisinistes, prévoient obligatoirement une double exclusivité : une exclusivité territoriale accordée au commerçant en contrepartie d'une exclusivité d'approvisionnement du commerçant auprès du concédant tête de réseau. Le contrat de franchise suppose un transfert de savoir-faire original et substantiel entre franchiseur et franchisé. Le contrat de commission-affiliation, qui devient la norme dans le prêt-à-porter, s'ajoute au contrat de franchise et prévoit, en outre, que le stock reste la propriété de la tête du réseau contractuel. Le coût et la gestion du stock ne sont donc pas supportés par le franchisé entendu comme affilié. Ces contrats ont fait l'objet du nouveau règlement d'exemption de la Commission des Communautés Européennes, entré en vigueur le $1^{\text {er }}$ juin 2010 pour onze ans. Ce règlement soustrait les accords verticaux à l'interdiction des ententes prévus par le Traité de Rome, lorsqu'ils améliorent l'efficience économique et sous réserve de ne pas contenir de restrictions caractérisées pour le distributeur (clauses noires comme des prix de vente minimaux imposés ou une restriction des ventes en ligne).

En tant que structure réticulaire, les réseaux contractuels représentent une alternative aux systèmes intégrés de la GDA, contrôlés par un petit nombre de groupes multinationaux. Ils permettent ainsi à un entrepreneur, qui a mis au point un concept de magasin distinctif, d'avoir directement accès au consommateur final. Forme organisationnelle originale, hydride entre le marché et la hiérarchie au sens de Williamson (1985), les réseaux contractuels reposent sur une collaboration étroite et interactive entre plusieurs entreprises, le franchiseur (ou le 
concédant), à qui revient généralement l'initiative du partenariat, et les franchisés (ou concessionnaires), commerçants indépendants aux plans juridique et financier, qui adhèrent à un réseau pour bénéficier d'un système de réussite commerciale : «Les franchiseurs sont censés fournir des lignes directrices en matière de gestion des opérations, de stratégies, de procédures, et de support marketing. Les franchisés doivent suivre ces lignes directrices et fournir au franchiseur les informations sur les ventes et les résultats d'exploitation, de sorte que les paiements de redevance (stipulés par contrat) soient exacts et exécutés en temps opportun. Les franchisés sont également invités à partager l'information opérationnelle pour aider le franchiseur à affiner ses efforts marketing » (Paswan et Wittmann, 2009: 177).

Le contrat repose sur une complémentarité des ressources et des compétences des partenaires, au sens de Wernerfelt (1984). Il répartit les rôles et les profits entre les deux entrepreneurs. La tête du réseau contractuel assure les fonctions de sélection des franchisés, de définition de la stratégie du réseau et de promotion de la marque. Il transmet au franchisé un savoir-faire éprouvé et exerce un contrôle des activités des commerçants indépendants, de manière à assurer l'homogénéité de l'offre visà-vis des clients. Le commerçant, quant à lui, est propriétaire de son fonds de commerce, il apporte ses moyens financiers, humains et managériaux au bénéfice du magasin. En bref, la franchise est clairement une activité entrepreneuriale fondée sur un projet de vie de la part du franchisé et un projet stratégique de la part du franchiseur; dans ce contexte singulier, les théories explicatives de la naissance, de la croissance et de la pérennisation de la franchise sont naturellement proches des courants liés à l'entrepreneuriat (Tuunanen et Hoy, 2007).

Désormais reconnus comme légitimes, les réseaux contractuels sont devenus une "norme organisationnelle » autorisant un développement rapide des magasins sur un territoire donné (Dumoulin et Gauzente, 2009). La direction bicéphale de l'activité (les décisions stratégiques relevant de la tête du réseau, les décisions sur le magasin relevant du commerçant indépendant), d'un côté, la motivation patrimoniale des commerçants, de l'autre, entraîneraient en outre une agilité organisationnelle et une meilleure réactivité des réseaux contractuels en cas de 
chocs externes, même s'ils ne sont pas toujours des entreprises de grande taille disposant d'importantes ressources. Certes, on compte parmi les franchiseurs quelques entreprises multinationales comme McDonald's, Speedy ou Carrefour, qui sont souvent des réseaux mixtes, s'appuyant à la fois sur des succursales et des magasins franchisés. Mais les têtes de réseaux (franchiseurs) sont le plus souvent des entreprises de taille plus modeste, de type PME, avec des équipes de quelques dizaines de salariés et des ressources limitées. Dans les deux cas, le chiffre d'affaires des franchiseurs est constitué des droits d'entrée dans le réseau contractuel payés par les commerçants indépendants, d'une redevance sur le chiffre d'affaires des magasins et, éventuellement, d'une marge commerciale sur les produits vendus par la centrale d'achat.

Logistique des réseaux contractuels : vers une mutualisation horizontale

Le franchiseur apporte dans la "corbeille de la mariée », outre une marque enseigne susceptible de rallier la clientèle, un concept de vente distinctif (assortiment et services, marketing mix et théâtralisation du magasin), mais aussi un savoir-faire qui fonde le contrat de franchise et rend l'activité du franchisé plus efficace. Au-delà des savoir-faire strictement codifiés et enseignés aux franchisés, par exemple en termes de méthodes de vente d'un parfum haut de gamme, de procédures standardisées de production d'un hamburger garantissant l'hygiène alimentaire ou de révision d'un véhicule automobile, le système de réussite proposé par le franchiseur s'appuie sur un ensemble de savoir-faire dits « organisationnels », au rang desquels figure sa capacité à gérer de manière performante la chaîne logistique au service de ses franchisés (Perrigot et al., 2009). La gestion du dernier kilomètre dans le tissu urbain repose notamment sur une expertise singulière du chauffeur livreur qui doit associer connaissances spatiales, connaissances matérielles et connaissances des usages de l'espace (Cholez, 2008), et le franchiseur capable de former ces professionnels dans des conditions optimales pourra espérer fidéliser plus aisément ses franchisés.

Il n'est donc pas surprenant que dans les réseaux contractuels, à l'image de la GDA, la logistique soit perçue, depuis de 
nombreuses années, comme une source d'avantage concurrentiel. Venant en soutien du processus d'achat, elle est organisée par le franchiseur dans le but d'optimiser la livraison vers les magasins. Il est ici possible de parler de mutualisation verticale dans la mesure où le franchiseur, en tant que pivot, met en place des infrastructures et des procédures logistiques à l'attention de son réseau. Il lui dédie une préparation de commandes commune qui irrigue du haut (entrepôt) vers le bas (magasins) l'ensemble du réseau. Certains réseaux contractuels ont ainsi créé des $C D$ pour leurs magasins et possèdent parfois leur propre flotte de camions (voir le cas La Vie Claire ci-après).

Les enjeux liés à une parfaite maîtrise de la logistique (et du management des achats) dans un réseau contractuel sont multiples. En termes d'efficacité économique et d'avantage concurrentiel, la logistique participe à la réussite du business model ; une modélisation récente des problématiques logistiques en contexte de franchise souligne ainsi leur impact sur les flux de trésorerie pour l'ensemble du réseau (Fenies et al., 2010). On retrouve les mêmes enjeux que dans la GDA : les achats groupés pour l'ensemble des magasins autorisent une puissance d'achat et un pouvoir de négociation accrus face aux fournisseurs, l'organisation logistique est une source d'économie de coûts avec un taux de service supérieur. Le contrôle de la chaîne logistique et de ses coûts de fonctionnement, une expertise clé pour le franchiseur, constitue pour lui une source de pouvoir au sens de French et Raven (1959). C'est une manière d'adresser un message clair à ses partenaires sur l'intérêt d'adhérer entièrement à son projet et ne pas être tentés de faire ponctuellement cavalier seul. Rappelons en effet que les franchisés, en tant que commerçants indépendants, conservent au final une certaine liberté d'approvisionnement auprès des industriels et/ou des grossistes, 1'exclusivité d'approvisionnement exigée par le franchiseur étant le plus souvent partielle.

Une logistique efficiente permet ainsi d'accroître la fidélisation des franchisés et leur taux d'approvisionnement auprès de la centrale d'achat. La logistique s'apparente ainsi clairement à une sorte de "catalyseur » du réseau contractuel qui évite la propagation d'effets centrifuges. Elle témoigne également de l'engagement $\mathrm{du}$ franchiseur dans la relation avec son (ses) franchisé(s), ce qui favorise l'adoption d'une logique de 
marketing relationnel (Watson et Johnson, 2010). En outre, la centralisation des commandes offre au franchiseur la possibilité d'accéder aux données de vente de chaque magasin et d'établir des comparaisons entre les commerçants indépendants selon une démarche de benchmarking. Un franchiseur peut donc être qualifié, à ce titre, de véritable PSL pour son réseau. Dans un article de Supply Chain Magazine de mai 2009, le Directeur général de La Vie Claire définit ainsi son métier de franchiseur par un raccourci saisissant : «Nous [La Vie Claire] sommes une entreprise de marketing et de logistique $»$.

$\mathrm{Au}$ total, on retrouve dans les réseaux contractuels les mêmes enjeux stratégiques et les mêmes défis, d'une part, de réduction des coûts, d'autre part, d'exercice d'un pouvoir d'influence, tels qu'identifiés dans la GDA. Qu'en est-il du troisième enjeu, de nature environnementale, qui pourrait conduire les parties prenantes à emprunter la voie de la mutualisation horizontale entre réseaux contractuels distincts? En effet, si l'objectif de réduction des coûts reste prégnant, avec les fortes hausses du prix de l'énergie, des pressions environnementales ne cessent de prendre de l'importance, sous l'influence de populations sensibilisées aux effets néfastes des activités logistiques. La réduction du nombre de kilomètres parcourus et l'optimisation du remplissage des camions deviennent des priorités en vue de réduire les émissions de $\mathrm{CO}_{2}$. La pression sociétale est particulièrement forte sur le commerce de centres ville, avec l'encombrement des rues piétonnes et les nuisances dues aux multiples camions de livraison. Les réseaux contractuels sont en première ligne pour réfléchir avec les managers des villes à ces aspects sensibles.

Dans ce contexte, ne peut-on pas imaginer, compte tenu de la taille des réseaux contractuels, que des expériences de mutualisation horizontale soient menées entre réseaux concurrents, avec des motivations diverses, qu'il s'agirait d'identifier? Plusieurs expériences commencent à être initiées en France, notamment des $\mathrm{CD}$ et des tournées de livraison communs à McDonald's, Courtepaille et Class'Croûte, trois entreprises de restauration qui n'ont aucun lien capitalistique et sont concurrentes même si elles ne sont pas exactement sur les mêmes positionnements; ces expériences confortent l'idée selon laquelle les évolutions observées dans la GDA pourraient s'éten- 
dre aux réseaux contractuels. Ceci ne doit pas faire passer sous silence un certain nombre de freins. Ainsi, convaincu de l'intérêt de la mutualisation des ressources logistiques, un franchiseur à la tête de plusieurs magasins dans le domaine des produits frais, rencontré en mars 2010 lors du Salon de la Franchise à Paris, nous indiquait avoir approché récemment ses concurrents directs dans le but de réfléchir à la mise en place de $C D$ partagés. Il s'est heurté à un refus ferme, ses concurrents arguant que l'exacerbation de la concurrence entre franchisés sur une même ville rend illusoire toute coopération en matière de logistique. Mais le simple fait qu'un franchiseur ait sollicité ses concurrents sur cette question montre que l'idée de mutualisation horizontale est en marche. Le cas La Vie Claire est un exemple instructif de mouvement des réseaux contractuels vers la mutualisation des ressources logistiques.

\section{La Vie Claire, une mutualisation réussie}

Avec 202 points de vente en France (dont 176 en franchise), La Vie Claire est le deuxième réseau de vente spécialisé en alimentation bio, après son concurrent Biocoop. Le franchiseur est une PME qui emploie plus de 200 salariés fin 2010. L'entreprise connaît à nouveau une forte croissance, avec un chiffre d'affaires de 53 millions d'Euros en 2009, en hausse de $21 \%$ par rapport à 2008, et un nombre d'ouverture de magasins soutenu (15 en 2009). Cela est permis grâce à la vitalité du marché bio, qui ne connaît guère la crise, mais aussi en raison du changement de concept distinctif de l'enseigne en 2003. L'internationalisation est également conduite à marche forcée puisque La Vie Claire s'est installée à Casablanca début 2011. Distribio et La Vie Claire Maroc, propriétaires de la franchise au Maroc, ont programmé d'ouvrir un deuxième point de vente à Casablanca et un troisième à Rabat, déjà en phase d'aménagement, d'ici fin 2011.

Créée en 1946, La Vie Claire a pourtant connu une histoire particulièrement mouvementée. D'abord, avec une stratégie marketing qui n'a pas toujours été favorable aux franchisés (ventes parallèles en hypermarchés). Ensuite, avec une succession de propriétaires: Bernard Tapie, ancien président de l'Olympique de Marseille, de 1980 à 1995, période au cours de 
laquelle le franchiseur acquiert une notoriété élevée et atteint 250 magasins franchisés; puis le groupe Distriborg, de 1996 à 2001, commercialisant par ailleurs en grandes surfaces les marques Bjorg, Vivis, Gayelord Hauser et Krisprolls notamment. En grande difficulté, le réseau contractuel ne compte plus que 80 magasins lorsqu'il est racheté en 2002 par le franchiseur actuel. Ce dernier opère un changement de concept avec, au cœur du dispositif, une organisation logistique verticale qui confère à l'enseigne un avantage concurrentiel clé.

Le nouveau concept prévoit une augmentation significative des surfaces de vente des magasins (de 50 à $300 \mathrm{~m}^{2}$ en moyenne) et une hausse importante du nombre de références : de 1200 en 2002, le nombre de références de produits bio atteint 4000 en 2010. Parmi elles, 1300 sont des marques de distributeurs (MDD) en fort développement (sous la marque La Vie Claire). L'objectif de l'organisation logistique est d'assurer des livraisons tous les jours, avant $8 \mathrm{~h} \mathrm{30}$, en $\mathrm{J}+1$ par rapport à la commande du magasin. Pour ce faire, La Vie Claire a acquis en 2002 un entrepôt de $4000 \mathrm{~m}^{2}$ à Chaponost (près de Lyon). Cet entrepôt ayant atteint ses limites, avec une taille insuffisante et un outil de préparation des commandes en fin de vie, un nouvel équipement de $13000 \mathrm{~m}^{2}$, à Montagny (Rhône), a été livré en décembre 2010, avec un financement de 13 millions d'euros assuré, pour 4 millions d'Euros, par l'ouverture du capital à NAXICAP. Par ailleurs, La Vie Claire a investi dans sa propre flotte de quatre véhicules pour les livraisons à Paris et en région parisienne.

Quatre chauffeurs travaillent la nuit de $22 \mathrm{~h}$ à $8 \mathrm{~h}$ car le franchiseur n'accepte pas les livraisons en journée. Comme l'indique le Directeur général de La Vie Claire, « la journée doit être dédiée au client et aussi pour des questions d'efficacité : le facteur efficacité est de un à trois entre le jour et la nuit pour ce qui concerne le nombre de magasins livrés, en raison des problèmes de circulation ». Les chauffeurs, qui possèdent les clés des magasins, les livrent en l'absence du franchisé, en respectant les zones de froid pour les produits délicats. Pour les autres régions, le transport est soit mutualisé avec une autre entreprise (une entreprise de fruits et légumes sur Lyon qui utilise les camions le jour alors que La Vie Claire les utilise la nuit), soit sous-traité à des transporteurs. C'est une première 
expérience de mutualisation horizontale pour La Vie Claire. L'objectif de ces investissements logistiques est clair : il s'agit de fidéliser les franchisés et de les amener à passer $100 \%$ de leurs commandes auprès de la centrale d'achat de La Vie Claire, alors même que le contrat de franchise leur laisse une liberté d'achat de $20 \%$ en dehors de ladite centrale. On peut affirmer que, dans ce cas, le franchiseur assume la fonction de PSL pour son réseau contractuel ${ }^{1}$.

\section{CONCLUSION}

La mutualisation des ressources logistiques constitue désormais une thématique majeure en vue d'améliorer le fonctionnement des chaînes logistiques, notamment dans la GDA et la GSS. Il s'agit ici d'une réelle rupture par rapport à la pensée dominante en supply chain management. En effet, pendant de nombreuses années, l'idée la plus courante, véhiculée notamment par les travaux de Christopher (2010), était de s'appuyer sur un modèle de compétition entre chaînes logistiques. La compétitivité résultait alors prioritairement d'une capacité des partenaires à structurer des relations de qualité le long d'une sorte de pipeline, du fournisseur du fournisseur au client du client. Cette vision linéaire sous-entend un cloisonnement relationnel entre chaînes logistiques selon une perspective verticale, qui définit la performance en termes de coût, de qualité de service et de réactivité à partir des échanges entre un industriel et un grand distributeur, ou un industriel, un PSL et un grand distributeur. Le modèle de la compétition « chaîne logistique vs chaîne logistique » découle ainsi directement du paradigme économique de la filière, héritier direct des analyses stratégiques en termes d'intégration verticale.

Les recherches initiées par Zhang et al. (2003) sur la «supply chain network economy» introduisent une vision radicalement nouvelle. Elles mettent l'accent sur l'interaction dynamique entre des acteurs appartenant simultanément à plusieurs chaînes logistiques; l'ensemble des chaînes logistiques

\footnotetext{
${ }^{1}$ Les informations sont tirées de la presse professionnelle et d'un entretien conduit par Odile Chanut avec le Directeur général de La Vie Claire, le 11 septembre 2009.
} 
constitue un réseau qui intègre un certain nombre de nœuds devant être simultanément performants pour plusieurs d'entre elles (par exemple, des CD ou des unités d'assemblage). La performance résulte alors de l'atteinte d'une taille critique dans le management des opérations dans chaque nœud, permettant la réalisation d'économies d'échelle et de champ. Dans le contexte de la "supply chain network economy», la mutualisation des ressources logistiques apparaît comme un levier opérationnel de première importance pour accroître l'efficacité des nœuds les plus critiques. Ceci est vrai pour les réseaux contractuels, c'est également vrai de façon plus générale pour l'ensemble de la GDA.

Il est incontestable pour la majorité des observateurs que les entreprises de la GDA ont su précocement initier des stratégies de mutualisation des ressources logistiques qui ont ouvert la voie à une application à d'autres formules de vente. L'assimilation s'est alors faite pas à pas selon un processus d'apprentissage organisationnel bien connu dans la littérature. La force des réseaux contractuels est toutefois d'avoir su s'émanciper en partie des cadres étroits d'optimisation pensés dans un contexte de grandes surfaces dont certaines sont localisées dans des espaces périurbains, et dont les schémas logistiques dominants reposent sur des logiques de massification extrême. Les réseaux contractuels s'ancrent au contraire dans un tissu commercial de proximité qui exige des adaptations techniques et managériales que certains franchiseurs ont été capables d'impulser. Il reste évidemment beaucoup à faire pour avancer dans notre connaissance des stratégies de mutualisation des ressources logistiques. Trois pistes de réflexion semblent ici importantes à explorer :

- Sur le plan des processus technologiques, la question est de savoir comment organiser au mieux la compatibilité des interfaces entre les différentes chaînes logistiques. Si la mutualisation des ressources logistiques conduit à mettre à disposition des moyens communs pour des membres d'une chaîne logistique en situation de concurrence frontale, encore faut-il qu'ils puissent partager ces moyens sans avoir à supporter des coûts d'ajustement exorbitants. Les interfaces doivent donc être suffisamment standardisées (ou génériques) pour autoriser la mutualisation des ressources logistiques. Il n'est pas sûr qu'un tel processus de standardisation pourra être mis 
rapidement en place, ni même accepté par les membres d'une chaîne logistique, jaloux de certaines de leurs prérogatives.

- Sur le plan des systèmes d'information, la mutualisation des ressources logistiques repose sur des informations de pilotage des flux dont le partage entre membres d'une chaîne logistique risque de poser problème (de Corbière et al., 2010). En effet, les informations de pilotage des flux intègrent des dimensions marketing que les membres d'une chaîne logistique voudront jalousement protéger. On sait par exemple que les réseaux contractuels souhaitent rapidement réagir aux campagnes promotionnelles des concurrents en lançant au plus vite leurs propres campagnes promotionnelles. Si les réseaux contractuels procèdent à une mutualisation des ressources logistiques, ils connaîtront dans de très brefs délais les actions marketing en préparation sur les CD communs, ce qui est risque d'annihiler tout politique de différenciation.

- Sur le plan des enjeux stratégiques, enfin, le risque est grand de voir se diluer la logistique comme source d'avantage concurrentiel entre tous les membres d'une chaîne logistique. En effet, la performance issue de la mutualisation des ressources logistiques dépend directement du potentiel d'activité que représentent le ou les partenaires, chaque partie tirant de l'autre partie les sources de son propre avantage concurrentiel. Ainsi, un réseau contractuel sera efficace sur le plan de la logistique parce qu'un réseau contractuel directement concurrent apporte un volume significatif d'affaires sur un ou plusieurs nœuds du «supply chain network», et vice versa. La mutualisation des ressources logistiques conduit ainsi à des comportements par nature mimétiques qui pourraient bloquer l'extension du processus dès lors qu'un réseau contractuel considèrera qu'il dispose d'un savoir-faire idiosyncrasique ne devant surtout pas être partagé avec des réseaux contractuels concurrents. 


\section{BIBLIOGRAPHIE}

ALBERTINI P., Les stratégies des prestataires logistiques européens, Paris, Éditions Eurostaf, 2008.

ASSENS C., 2003, "Le réseau d'entreprises : vers une synthèse des connaissances ", Management International, vol. 7, $\mathrm{n}^{\circ} 4$, p. 49-59.

BOULAY J., CHANUT O., Les réseaux de franchise, Paris, La Découverte, 2010.

CHOLEZ C., "Compétences spatiales, compétences d'action dans l'espace : la tournée du chauffeur-livreur », Revue d'Anthropologie des Connaissances, vol. 2, n 1, 2008, p. 37-62.

CHRISTOPHER M., Logistics and supply chain management, Harlow, FT Prentice Hall, $4^{\mathrm{e}}$ éd. 2010.

DE CORBIERE F., DURAND B., ROWE F., «Effets économiques et environnementaux de la mutualisation des informations logistiques de distribution: avis d'experts et voies de recherche " , Management \& Avenir, $\mathrm{n}^{\circ} 39,2010$, p. 326-348.

DUMOULIN R., GAUZENTE C., «Les facteurs d'institutionnalisation de la franchise et leurs conséquences sur la performance », Management \& Avenir, $\mathrm{n}^{\circ} 22,2009$, p. 155-170.

FENIES P., LAGRANGE S., TCHERNEV N., "A decisional modelling for supply chain management in franchised networks: application in franchise bakery networks", Production Planning \& Control, vol. 21, n 6, 2010, p. 595-608.

FRENCH J., RAVEN B., "The bases of social power", in D. Cartwright (sous la direction de), Studies in social power, Ann Arbor (MI), University of Michigan Press, 1959, p. 150-167.

HIESSE V., PACHÉ G., "Logistique mutualisée : une nouvelle architecture organisationnelle dans les canaux de distribution », Économies et Sociétés, série Systèmes agroalimentaires, $\mathrm{n}^{\circ} 32$, 2010, p. 1513-1533.

LAFONTAINE F., "The evolution of franchising and franchise contracts: evidence from the United States", Entrepreneurial Business Law Review, vol. 3, n 2, 2009, p. 381-434.

PACHÉ G., «Penser la décision en sciences de gestion : pour une vision de nature syncrétique ", in J.-R. Alcaras, P. Gianfaldoni et G. Paché (sous la direction de), Décider dans les organisations, Paris, L'Harmattan, 2004, p. 79-99. 
PACHÉ G., «Logistique urbaine mutualisée : quelle stratégie de différenciation pour le commerce alimentaire en ligne? ", Revue française de gestion industrielle, vol. 29, n 2, 2010, p. 27-47.

PAN S., Contribution à la définition et à l'évaluation de la mutualisation de chaînes logistiques pour réduire les émissions de $\mathrm{CO}_{2}$ du transport : application au cas de la grande distribution, Thèse de doctorat en Sciences de Gestion, École nationale supérieure des Mines de Paris, décembre, 2010.

PAN S., BALLOT É., FONTANE F., "The reduction of greenhouse gas emissions from freight transport by pooling supply chains", International Journal of Production Economics, 2011 (à paraître).

PASWAN A., WITTMANN, C., "Knowledge management and franchise systems", Industrial Marketing Management, vol. 38, $\mathrm{n}^{\circ} 2,2009$, p. 173-180.

PERRIGOT R., EL AKREMI A., HERRBACH O., MIGNONAC $\mathrm{K}$., Quels sont les savoir-faire sources de l'avantage concurrentiel en franchise? Paris, Fédération Française de la Franchise, 2009.

PIPAME (Pôle interministériel de prospective et d'anticipation des mutations économiques), Logistique mutualisée : la filière "fruits \& légumes » du Marché d'Intérêt National de Rungis, Paris, ministère de l'Économie, de l'Industrie et de l'Emploi, 2009.

RAJESH R., PUGAZHENDHI S, GANESH K., "Towards taxonomy architecture of knowledge management for third party logistics service provider", Benchmarking: An International Journal, vol. $18, n^{\circ} 1,2011$, p. $42-68$.

TUUNANEN M., HOY F., "Franchising-multifaceted form of entrepreneurship", International Journal of Entrepreneurship and Small Business, vol. 4, $\mathrm{n}^{\circ}$ 1, 2007, p. 52-67.

WATSON A., JOHNSON R., "Managing the franchisor-franchisee relationship: a relationship marketing perspective", Journal of Marketing Channels, vol. 17, $\mathrm{n}^{\circ}$ 1, 2010, p. 51-68.

WERNERFELT B., "The resource-based view of the firm", Strategic Management Journal, vol. 5, n 2, 1984, p. 171-180.

WILLIAMSON O., 1985, The economic institutions of capitalism : firms, market, relational contracting, New York (NY), The Free Press, 1985.

YAMI S., LE ROY F. (sous la direction de), Stratégies de coopétition : rivaliser et coopérer simultanément, Bruxelles, De Boeck, 2010.

ZHANG D., DONG J., NAGURNEY A., “A supply chain network economy: modeling and qualitative analysis", in A. Nagurney 
(sous la direction de), Innovations in financial and economic networks, Cheltenham, Edward Elgar Publishing, 2003, p. 197213. 